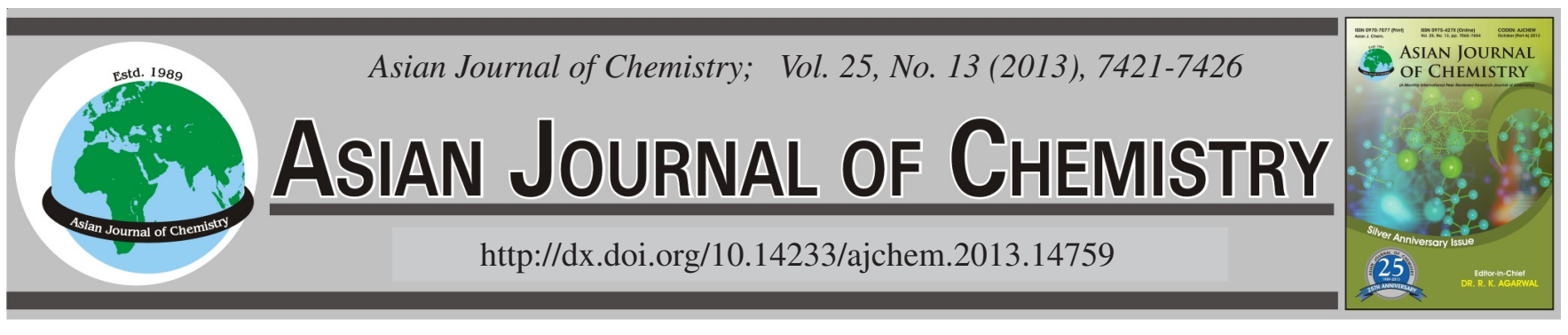

\title{
Selective Adsorption of Berberine Hydrochloride Using Molecularly Imprinted Polymers with Modified Rosin as Cross-linker
}

\author{
Hao Li ${ }^{1,2}$, Fuhou Lei ${ }^{1,2, *}$, Pengfei Li ${ }^{1,2}$, Wengui Duan ${ }^{3}$, Juying Zhou $^{1,2}$ and Xuecai Tan ${ }^{1,2}$
}

${ }^{1}$ School of Chemistry and Chemical Engineering, Guangxi University for Nationalities, Nanning 530006, P.R. China

${ }^{2}$ Guangxi Key Laboratory of Chemistry and Engineering of Forest Products, Nanning 530006, P.R. China

${ }^{3}$ School of Chemistry and Chemical Engineering, Guangxi University, Nanning 530004, P.R. China

*Corresponding author: Fax: +86 771 3261718; Tel: +86 771 3262198; E-mail: leifuhou@gmail.com

(Received: 1 November 2012;

Accepted: 29 June 2013)

AJC-13720

\begin{abstract}
Molecularly imprinted polymers containing phenanthrene skeleton were prepared via suspension polymerization with ethylene glycol maleic rosinate acrylate (EGMRA) as a cross-linker, berberine hydrochloride as the template and methacrylic acid as the functional monomer. The binding characteristics of the molecularly imprinted polymers and their templates were evaluated via equilibrium binding, whereas those of berberine hydrochloride on molecularly imprinted polymers were evaluated via Scatchard analysis. The adsorption energy was measured via microcalorimetry, whereas the selective recognition of molecularly imprinted polymers was investigated through high-performance liquid chromatography. Scatchard plot analysis reveals the formation of two types of binding sites in the molecularly imprinted polymers, with their respective equilibrium dissociation constants at $\mathrm{K}_{\mathrm{d} 1}=0.0204$ and $\mathrm{K}_{\mathrm{d} 2}=0.0507 \mathrm{mmol} \cdot \mathrm{L}^{-1}$. The heat flow curve exhibits an endothermic peak and two relatively weak exothermic peaks for the adsorption. Selective adsorption results demonstrate the high affinity and berberine hydrochloride selectivity of the molecularly imprinted polymers.
\end{abstract}

Key Words: Berberine hydrochloride, Molecularly imprinted polymers, Selective adsorption, Microcalorimetry, Modified rosin.

\section{INTRODUCTION}

Molecular imprinting is becoming increasingly recognized as a powerful technique of preparing synthetic polymers that contain tailor-made recognition sites for target molecules ${ }^{1}$. The preparation of molecularly imprinted polymers involves the formation of complexes between functional monomers and a template molecule in the appropriate solution; the composites are fixed via polymerization at a high cross-linker ratio ${ }^{2,3}$. The subsequent removal of the template molecule leaves cavities that exhibit the size, shape and arrangement of functional groups that are complementary to the template molecule ${ }^{4}$.

In recent years, molecularly imprinted polymers with high selectivity and good stability have been widely used in solid phase extraction $^{5-7}$, membrane separation ${ }^{8}$ and chiral separation $^{9,10}$, as well as in catalyst ${ }^{11}$ and bionic sensor ${ }^{12,13}$.

The synthesis of molecularly imprinted polymers usually involves bulk polymerization ${ }^{14}$. The products obtained are usually bulky and require grinding or sieving prior to use. Grinding is not only time-consuming, it destroys some of the binding sites and reduces the molecular recognition ability and selectivity of the material ${ }^{15}$. In this work, the polymer was obtained through the suspension polymerization process, yielding products with an even particle size and thus requiring no grinding.

Generally, molecularly imprinted polymers were prepared using ethylene glycol dimethacrylate ${ }^{16}$ as a cross-linker. The use of ethylene glycol dimethacrylate as the cross-linker results in the poor rigidity of molecularly imprinted polymers and leads to irreversible distortion of the molecularly imprinted holes. These characteristics affect the recognition performance and selectivity of the molecularly imprinted polymers. To address these challenges, considerable effort has been focused on improving the mechanical strength and chemical stability of molecularly imprinted polymers. Thus, the development a novel cross-linker is important for the synthesis of molecularly imprinted polymers with high selectivity and stability.

In this work, ethylene glycol maleic rosinate acrylate (EGMRA; Fig. 1) was used as the ligand for affinity separation by introducing the phenanthrene skeleton into the polymers to improve their mechanical strength and stability. The molecularly imprinted polymers were prepared using ethylene glycol maleic rosinate acrylate as the novel cross-linker, berberine hydrochloride as the template and methacrylic acid as the functional monomer. The characterization of the obtained particles was performed through scanning electron microscopy. The 
adsorption properties of molecularly imprinted polymers were investigated via equilibrium adsorbing experiments and the Scatchard analysis. The quantity of heat adsorbed was determined via the microcalorimetric method. The selectivity of the obtained particles was elucidated from the different binding abilities of berberine hydrochloride and ligustrazine hydrochloride.

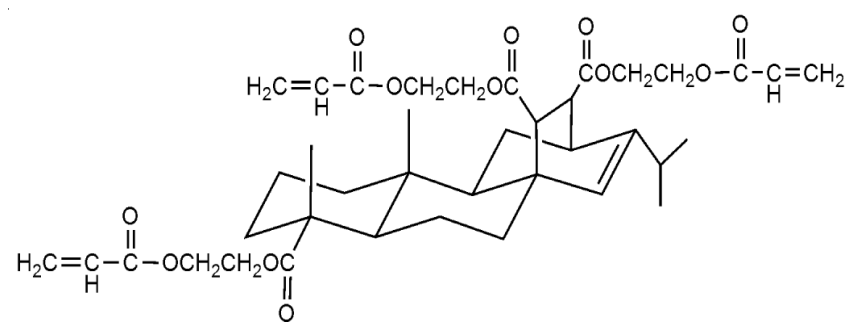

Fig. 1. Structure of the novel cross-linker, ethylene glycol maleic rosinate acrylate (EGMRA)

\section{EXPERIMENTAL}

Berberine hydrochloride and ligustrazine hydrochloride were purchased from Yuxin Pharmaceutical Co. Ltd., (Sichuan, China). Ethylene glycol maleic rosinate acrylate was prepared in our laboratory. Methacrylic acid, sodium dodecyl sulfate, glycol, glacial acetic acid, anhydrous alcohol, methanol, azodiisobutyronitrile and sodium hydroxide were all obtained from Xilong Chemical Reagent Co. Ltd., (Shantou, China). The methanol used was high-performance liquid chromatography (HPLC)-grade, whereas all other reagents were analytical-grade.

Molecularly imprinted polymers and non-imprinted polymers preparation: The granular polymers were synthesized as follows: $49 \mathrm{mmol}$ methacrylic acid monomer and 14 mmol ethylene glycol maleic rosinate acrylate cross-linker were dissolved in $30 \mathrm{~mL}$ ethyl acetate, while $1.4 \mathrm{mmol}$ berberine hydrochloride template was dissolved in $20 \mathrm{~mL}$ glycol. The solutions were then mixed in a $500 \mathrm{~mL}$ glass flask. Azodiisobutyronitrile $(0.35 \mathrm{~g})$ and porogen $(8 \mathrm{~mL})$ were dissolved in the solution via ultrasonication. Sodium dodecyl sulfate $(0.10 \mathrm{~g})$ and distilled water $(250 \mathrm{~mL})$ were then added into the solution. The mixture was predispersed at $400 \mathrm{rpm}$ for $10 \mathrm{~min}$ at room temperature using a JB300-D agitator (Shanghai, China). The reaction was then maintained at $95^{\circ} \mathrm{C}$ for $1.5 \mathrm{~h}$. Finally, the obtained polymers were washed with a methanol and acetic acid solution $(4: 1 \mathrm{v} / \mathrm{v})$ to remove the template molecules.

The non-imprinted polymers were prepared using the same method but without the addition of the templates.

For comparison, the polymers were also synthesized with ethylene glycol dimethacrylate as the cross-linker following the same protocol.

Scanning electron microscopy: The morphologies of the obtained polymers were determined via a SEM (Hitachi S-3400 N, Japan). Prior to visualization, a small section of the polymer was placed on the SEM sample holder and was then sputter-coated with gold ${ }^{17}$.

Adsorption experiment: Adsorption was performed at $30{ }^{\circ} \mathrm{C}$ in a shaker operated at $100 \mathrm{rpm}$. Two kinds of adsorption experiments were performed. In the kinetic binding experiments, $2.45 \mathrm{mmol} \mathrm{L}^{-1}$ berberine hydrochloride solution was used, whereas in the isotherm experiments, the initial berberine hydrochloride concentration ranged from $0.5 \mathrm{mmol}$ $\mathrm{L}^{-1}$ to $5.5 \mathrm{mmol} \mathrm{L}^{-1}$. The berberine hydrochloride concentration in the solution was determined using a UV-752 spectrophotometer at $345 \mathrm{~nm}$. The binding amount Qt $\left(\mathrm{mmol} \mathrm{g}^{-1}\right)$ was calculated from the following equation ${ }^{18}$ :

$$
\mathrm{Q}_{\mathrm{t}}=\frac{\mathrm{V} \times\left(\mathrm{C}_{0}-\mathrm{C}_{\mathrm{t}}\right)}{\mathrm{m}}
$$

where $\mathrm{C}_{0}$ is the initial berberine hydrochloride concentration $\left(\mathrm{mmol} \mathrm{L}{ }^{-1}\right), \mathrm{C}_{\mathrm{t}}$ is the berberine hydrochloride concentration $\left(\mathrm{mmol} \mathrm{L}{ }^{-1}\right)$ at different time points $\mathrm{t}, \mathrm{V}$ is the volume of the berberine hydrochloride solution $(\mathrm{mL})$ and $\mathrm{m}$ is the molecularly imprinted polymers/non-imprinted polymers weight $(\mathrm{g})$.

Microcalorimetric experiment: The energy evolution was measured using a microcalorimeter (RD496-CK2000, Mian Yang CP Thermal Analysis Instrument Co. Ltd., China). The microcalorimeter was first calibrated using the Joule effect. The sensitivities of the calorimeter were determined as $(64.54$ $\pm 0.02),(64.56 \pm 0.03),(64.60 \pm 0.01),(64.68 \pm 0.02)$ and $(64.84 \pm 0.01) \mu \mathrm{V} \mathrm{mW} \mathrm{mW}^{-1}$ at $297.15,298.15,301.15,303.15$ and $308.15 \mathrm{~K}$, respectively. The electric power $(100 \mathrm{~mW})$ was provided to the Joule cell, which had $1000 \Omega$ electrical resistance for $100 \mathrm{~s}$ at $298.15 \mathrm{~K}$. The enthalpy of dissolution of $\mathrm{KCl}$ (spectral purity grade) in deionized water was (17.237 \pm $0.046) \mathrm{kJ} \mathrm{mol}^{-1}$, which is consistent with an earlier measurement $^{19}$ of $(17.241 \pm 0.018) \mathrm{kJ} \mathrm{mol}^{-1}$. The calorimetric system was accurate (accuracy of $0.02 \%$ ) and reliable (precision of $0.3 \%)$.

Energy changes in the berberine hydrochloride adsorption were detected at $298.15 \mathrm{~K}$. The berberine hydrochloride concentration and volume were $2.45 \mathrm{mmol} \mathrm{L}^{-1}$ and $2 \mathrm{~mL}$, respectively. The mass of the molecularly imprinted polymers was $0.008 \mathrm{~g}$.

Regeneration experiments: A certain amount $(0.2 \mathrm{~g})$ of molecularly imprinted polymers was added to a $50 \mathrm{~mL}$ of 2.45 mmol L ${ }^{-1}$ berberine hydrochloride solution for $3.5 \mathrm{~h}$ for berberine hydrochloride binding. The molecularly imprinted polymers were then regenerated in $50 \mathrm{~mL}$ of a $1 \mathrm{wt} \% \mathrm{HCl} \cdot \mathrm{C}_{2} \mathrm{H}_{5} \mathrm{OH}$ mixture in a thermostatic shaker at $30 \cdot \mathrm{C}$ for $3.5 \mathrm{~h}$ and were then washed with distilled water. The regenerated molecularly imprinted polymers were dried at room temperature and reused in the subsequent cycle of rebinding experiments ${ }^{20}$.

HPLC: The HPLC system (Waters 1500-Series, America) consisted of a Waters 1525 Binary HPLC Pump and a Waters 2489 UV/visible detector. Chromatographic separation was performed on a chromatographic column (him-pack PREPODS(H) Kit; $5 \mu \mathrm{m} ; 250 \mathrm{~mm} \times 4.6 \mathrm{~mm}$ ) using a methanol mobile phase at a flow rate of $2 \mathrm{~mL} \mathrm{~min}^{-1}$. The column temperature was set at $30{ }^{\circ} \mathrm{C}$ and the injection volume was $20 \mu \mathrm{L}$. The detection wavelengths were set at 298 and $345 \mathrm{~nm}$. All samples were filtered using a $0.25 \mu \mathrm{m}$ microfilter prior to injection into the column system.

Treatment of berberine pill: $250 \mathrm{mg}$ of berberine pill power was mixed with $250 \mathrm{~mL}$ of distilled water and then ultrasonicated for $0.5 \mathrm{~h}$ at room temperature. The mixture was centrifuged at $3000 \mathrm{rpm}$ for $10 \mathrm{~min}$ and the supernatant was collected and applied to the molecularly imprinted polymer- 
SPE. The final solution was diluted 10 times with methanol and then analyzed by HPLC.

\section{RESULTS AND DISCUSSION}

Preparation of imprinted polymers: In this work, the molecularly imprinted polymers for berberine hydrochloride were prepared via the suspension polymerization method using berberine hydrochloride, ethylene glycol maleic rosinate acrylate (or EGDMA) and methacrylic acid as the template molecule, cross-linker and functional monomer, respectively. The granular polymers were prepared with ethylene glycol maleic rosinate acrylate as the cross-linker, whereas the powdered polymers were synthesized using ethylene glycol dimethacrylate. Furthermore, the adsorption quantities of berberine hydrochloride in the two polymers are significantly different (Table-1). This difference may be due to the use of different cross-linkers. Polymers prepared with ethylene glycol dimethacrylate as the cross-linker exhibited low mechanical strength. Moreover, their molecularly imprinted holes were irreversibly distorted and their recognition and selectivity were reduced. On the other hand, ethylene glycol maleic rosinate acrylate contains the phenanthrene skeleton (Fig. 1), which can stabilize the imprinted binding sites and impart mechanical stability to the polymer matrix. Thus, the molecularly imprinted polymers and non-imprinted polymers for the subsequent experiments were prepared with ethylene glycol maleic rosinate acrylate as the cross-linker.

\begin{tabular}{|c|c|c|}
\hline \multicolumn{3}{|c|}{$\begin{array}{l}\text { TABLE-1 } \\
\text { BINDING AMOUNT OF THE PREPARED POLYMERS } \\
\text { WITH DIFFERENT CROSS-LINKERS }\end{array}$} \\
\hline Polymers & Cross-linker & Adsorption $\left(\mathrm{mmol} \mathrm{g}^{-1}\right)$ \\
\hline 1\#MIPs ${ }^{\mathrm{a}}$ & EGMRA $^{\mathrm{c}}$ & 0.6245 \\
\hline $2 \# N I P s{ }^{b}$ & EGMRA $^{\mathrm{c}}$ & 0.3576 \\
\hline 3\#MIPs ${ }^{\mathrm{a}}$ & EGDMA $^{\mathrm{d}}$ & 0.1117 \\
\hline $4 \# N I P s^{b}$ & EGDMA $^{\mathrm{d}}$ & 0.1192 \\
\hline
\end{tabular}

The morphologies of the molecularly imprinted polymers and non-imprinted polymers were determined via SEM (Fig. 2). The SEM images reveal the loose structure of molecularly imprinted polymers particle, as well as the presence of numerous cavities and channels on the particle surfaces (Fig. 2a). By contrast, the non-imprinted polymers structure was compact (Fig. 2b). This difference probably resulted from the different template and porogen molecules used in the polymerization.
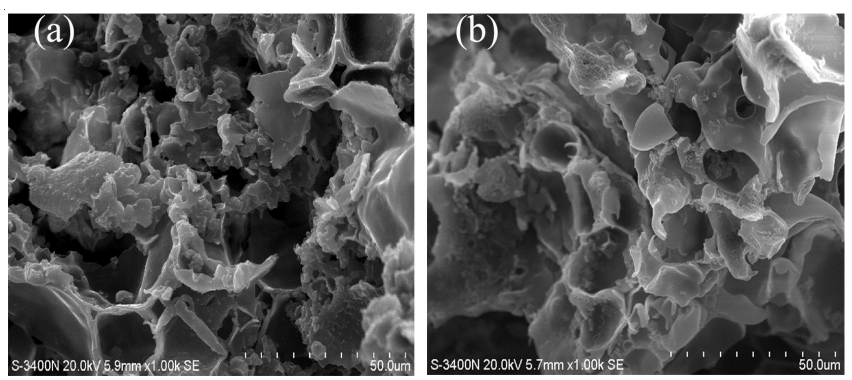

Fig. 2. Scanning electron microscope images of MIPs (a) and NIPs (b)
Dynamics adsorption of berberine hydrochloride on molecularly imprinted polymers: Fig. 3 shows the dynamic curve for the berberine hydrochloride adsorption onto the molecularly imprinted polymer particles. The amount of berberine hydrochloride adsorbed by the molecularly imprinted polymers sharply increased at $1 \mathrm{~h}$; the adsorption rate then decreased and the adsorption equilibrium was achieved at approximately $3.5 \mathrm{~h}$. The results demonstrate that the berberine hydrochloride molecules were preferentially adsorbed on the molecularly imprinted polymer surface sites and that the adsorption rate was faster at the start of adsorption. After full adsorption of berberine hydrochloride onto the molecularly imprinted polymer surfaces, the combination sites for the berberine hydrochloride molecules and molecularly imprinted polymer internal holes were blocked, consequently reducing the adsorption rate of the molecularly imprinted polymers.

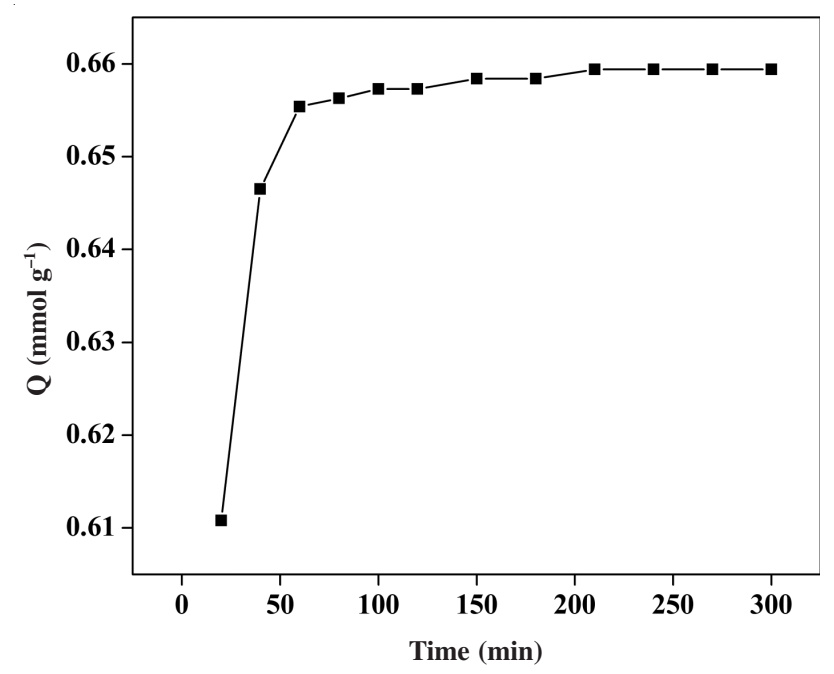

Fig. 3. Adsorption dynamic curve of molecularly imprinted polymers for berberine hydrochloride solution

Binding isotherm and Scatchard analysis: The binding characteristics of the molecularly imprinted polymers were determined via static adsorption experiments. The isotherms of berberine hydrochloride adsorption on the molecularly imprinted polymers were determined at the initial berberine hydrochloride concentrations (from $0.5 \mathrm{mmol} \mathrm{L}^{-1}$ to $5.5 \mathrm{mmol}$ $\left.\mathrm{L}^{-1}\right)$. The results are shown in Fig. 4a. Fig. 4a shows that as the berberine hydrochloride concentration increased, the adsorption capacity of molecularly imprinted polymers also increased. The adsorption quantity showed no changes at lower berberine hydrochloride concentrations. The difference between the adsorption capacities of molecularly imprinted polymers and non-imprinted polymers gradually increased when the berberine hydrochloride concentration exceeded $1.0 \mathrm{mmol} \mathrm{L}^{-1}$. These results demonstrate the remarkable adsorption ability of molecularly imprinted polymers for berberine hydrochloride.

The adsorption characteristics of the molecularly imprinted polymers were evaluated using the Scatchard model. The Scatchard equation ${ }^{20,21}$ is as follows:

$$
\frac{\mathrm{Q}}{\mathrm{C}}=\frac{\mathrm{Q}_{\max }-\mathrm{Q}}{\mathrm{K}_{\mathrm{d}}}
$$


where $\mathrm{Q}$ is the adsorption capacity at equilibrium, $\mathrm{C}$ is the berberine hydrochloride equilibrium concentration, $\mathrm{Q}_{\max }$ is the maximum apparent amount of the binding sites and $\mathrm{K}_{\mathrm{d}}$ is the equilibrium dissociation constants of the binding sites.

The Scatchard plot is shown in Fig. 4b. The plot exhibits two straight lines with a good linear relationship. The Scatchard analysis shows that the two different types of binding sites are located between the molecularly imprinted polymers and berberine hydrochloride molecule ${ }^{21}$. This phenomenon is due to the heterogeneity of the noncovalent complexes between the template molecule and the functional monomer in the molecularly imprinted polymers ${ }^{22}$. The linear eqns. 3 and 4 were obtained through linear fitting of the experimental data, as follows:

$$
\begin{aligned}
\mathrm{Q} / \mathrm{C} & =33.7453-49.012 \mathrm{Q} \\
\mathrm{Q} / \mathrm{C} & =18.4653-197136 \mathrm{Q}
\end{aligned}
$$

The $\mathrm{Q}_{\max }$ and $\mathrm{K}_{\mathrm{d}}$ were calculated from the intercept and slope of the straight line. The $\mathrm{Q}_{\max 1}$ and $\mathrm{K}_{\mathrm{d} 1}$ of the high-affinity binding sites are $0.688 \mathrm{mmol} \mathrm{g}^{-1}$ and $0.0204 \mathrm{mmol} \mathrm{L}^{-1}$, respectively, whereas $\mathrm{Q}_{\max 2}$ and $\mathrm{K}_{\mathrm{d} 2}$ of the low-affinity binding sites are $0.936 \mathrm{mmol} \mathrm{g}^{-1}$ and $0.0507 \mathrm{mmol} \mathrm{L}^{-1}$, respectively.
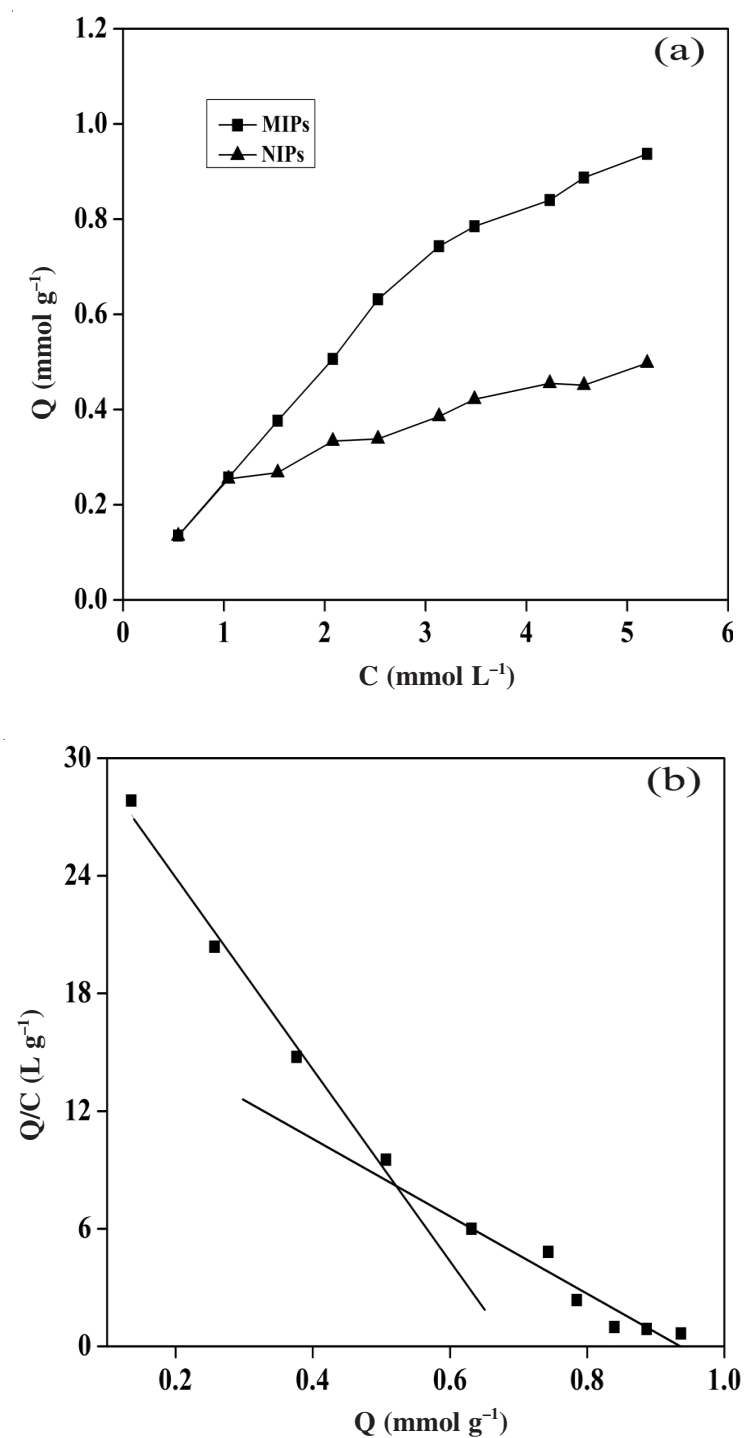

Fig. 4. Isotherms of BH adsorption on MIPs and NIPs (a); Scatchard plot for BH adsorption on MIPs (b)
Microcalorimetric studies on the adsorption process: The heat flow curve for the molecularly imprinted polymer adsorption was obtained using a RD496-CK2000 microcalorimeter at $298.15 \mathrm{~K}$ (Fig. 5). After an initial thermal disturbance caused by the loading of the stainless steel sample cells, a flat segment $\left(t_{1}=0-3491 \mathrm{~s}\right)$ that indicates the occurrence of negligible thermal events was observed. The curve during this period was taken as the initial baseline for the succeeding thermal peaks.

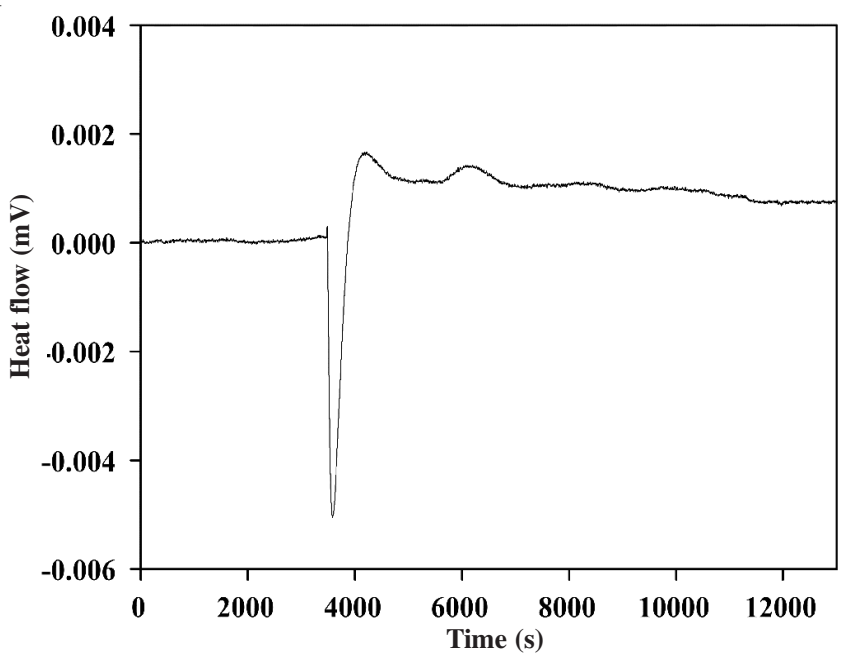

Fig. 5. Heat flow curve for the adsorption at $298.15 \mathrm{~K}$

The curve shows one strong endothermic peak and two relatively weak and discontinuous exothermic peaks. The reaction was initiated by the mixing of the molecularly imprinted polymers with the berberine hydrochloride solution. The energy sharply decreased, as indicated by the single subsequent endothermic peak in the heat flow curve. After approximately $90 \mathrm{~s}\left(\mathrm{t}_{2}=3581 \mathrm{~s}\right)$, an endothermic maximum was reached. Thereafter, the endothermic event rapidly changed into a correspondingly more pronounced exothermic event. At $\mathrm{t}_{3}=1.25 \mathrm{~h}$, the heat flow curve crossed the baseline (taken as the extension of the initial baseline) from endothermic to exothermic. At $t_{3}=4180 \mathrm{~s}$, an exothermic peak appeared. After approximately $0.55 \mathrm{~h}\left(\mathrm{t}_{4}=6145 \mathrm{~s}\right)$, the second exothermic peak was observed. At $12000 \mathrm{~s}$, the microcalorimetric curve flattened and remained unchanged for a long period. Thereafter, this flat curve served as the final baseline for the preceding thermal peaks. These results indicate that the berberine hydrochloride adsorption on molecularly imprinted polymers is a complicated exothermic process.

Molecularly imprinted polymer regeneration: Table- 2 shows the adsorptivity of the molecularly imprinted polymers for berberine hydrochloride, as determined via 20 consecutive adsorption experiments. The results showed that the molecularly imprinted polymers could be used many times and maintained their adsorption capacity at an almost constant value.

Molecularly imprinted polymer selectivity: The selectivity of molecularly imprinted polymers was investigated by using a mixed solution of berberine hydrochloride and ligustrazine hydrochloride (both $2.45 \mathrm{mmol} \mathrm{L}^{-1}$ ) at optimum adsorption conditions. The berberine hydrochloride and 
TABLE-2

ADSORPTION CAPACITY OF THE MIPs ${ }^{\mathrm{a}}$ FOR BERBERINE HYDROCHLORIDE ${ }^{\mathrm{b}}$ IN 20 CONSECUTIVE BINDING REACTIONS

\begin{tabular}{cc|cc|cc|cc}
\hline $\begin{array}{c}\text { Cycle } \\
\text { number }\end{array}$ & $\begin{array}{c}\text { Adsorption } \\
\left(\mathrm{mmol} \mathrm{g}^{-1}\right)\end{array}$ & $\begin{array}{c}\text { Cycle } \\
\text { number }\end{array}$ & $\begin{array}{c}\text { Adsorption } \\
\left(\mathrm{mmol} \mathrm{g}^{-1}\right)\end{array}$ & $\begin{array}{c}\text { Cycle } \\
\text { number }\end{array}$ & $\begin{array}{c}\text { Adsorption } \\
\left(\mathrm{mmol} \mathrm{g}^{-1}\right)\end{array}$ & $\begin{array}{c}\text { Cycle } \\
\text { number }\end{array}$ & $\begin{array}{c}\text { Adsorption } \\
\left(\mathrm{mmol} \mathrm{g}^{-1}\right)\end{array}$ \\
\hline 1 & 0.615 & 6 & 0.615 & 11 & 0.616 & 16 & 0.606 \\
2 & 0.603 & 7 & 0.607 & 12 & 0.613 & 17 & 0.599 \\
3 & 0.587 & 8 & 0.608 & 13 & 0.608 & 18 & 0.603 \\
4 & 0.584 & 9 & 0.612 & 14 & 0.620 & 19 & 0.595 \\
5 & 0.607 & 10 & 0.625 & 15 & 0.600 & 20 & 0.602 \\
\hline
\end{tabular}

ligustrazine hydrochloride concentrations in the mixture were detected via HPLC; the results are shown in Fig. 6.
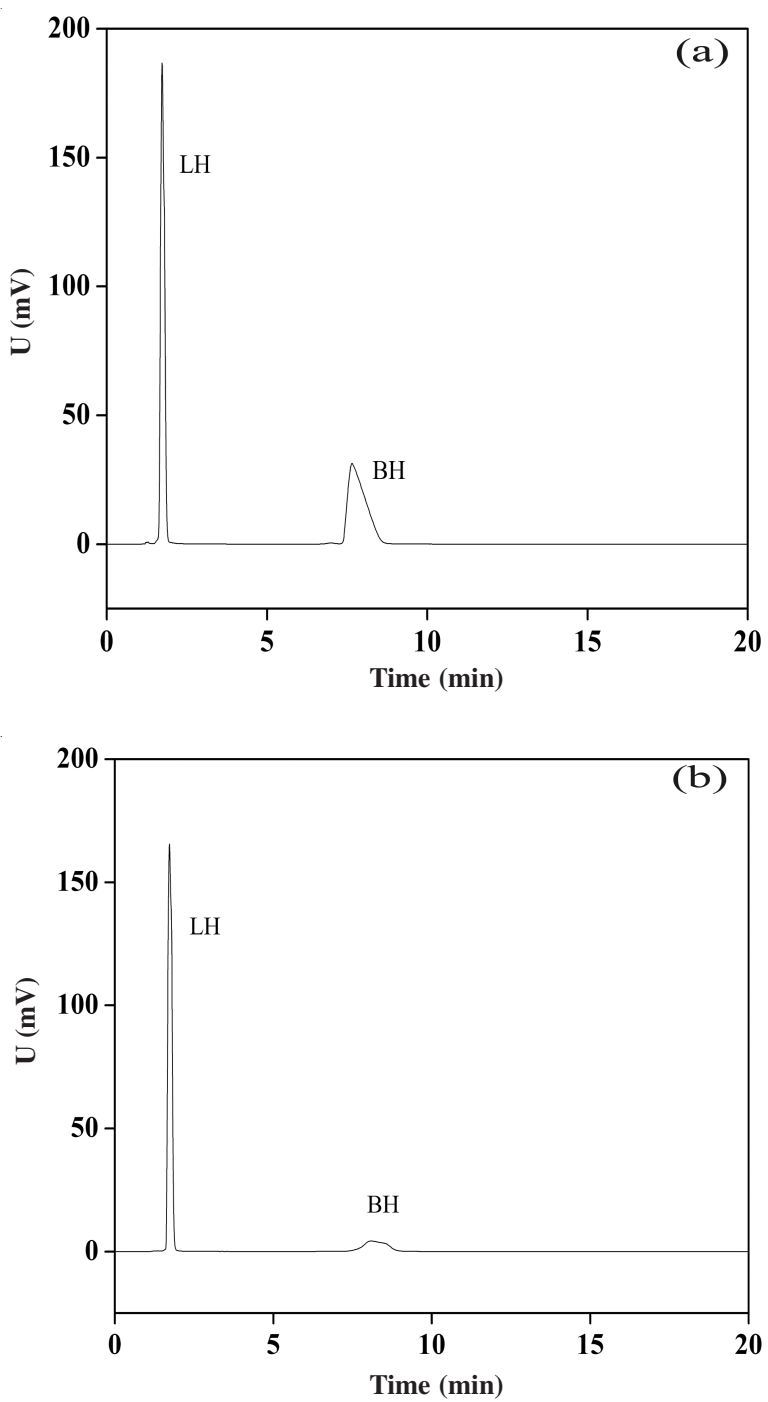

Fig. 6. HPLC chromatogram for the base solution before (a) and after (b) adsorption

The adsorption peak area at pre- and post adsorption significantly changed (shown in Fig. 6a and 6b, respectively) and the berberine hydrochloride content decreased by $83.5 \%$. However, the ligustrazine hydrochloride content decreased only by $6.52 \%$. These results demonstrate the remarkable selectivity of molecularly imprinted polymers for berberine hydrochloride in the presence of another substrate.

Analytical application: In order to evaluate the capacity of the molecularly imprinted polymer in practical application, the polymers as sorbent were applied in a complex berberine sample. The berberine pill was treated as described above. 0.1 $\mathrm{g}$ of molecularly imprinted polymer was added to a $25 \mathrm{~mL}$ of berberine sample solution for $3.5 \mathrm{~h}$, then the molecularly imprinted polymer was completely eluted using $25 \mathrm{~mL}$ of a $1 \mathrm{wt} \% \mathrm{HCI} \cdot \mathrm{C}_{2} \mathrm{H}_{5} \mathrm{OH}$ solution. The sample solution and elution solution were analyzed with HPLC. The results were shown in Fig. 7. The peaks that appeared at $7.54 \mathrm{~min}$ (Fig. 7a) and $7.58 \mathrm{~min}$ (Fig. 7b) are ascribed to berberine hydrochloride. As the result shown, berberine hydrochloride was strongly and selectively extracted by the molecularly imprinted polymer. And the molecularly imprinted polymer-SPE extraction recovery was $80.1 \%$.
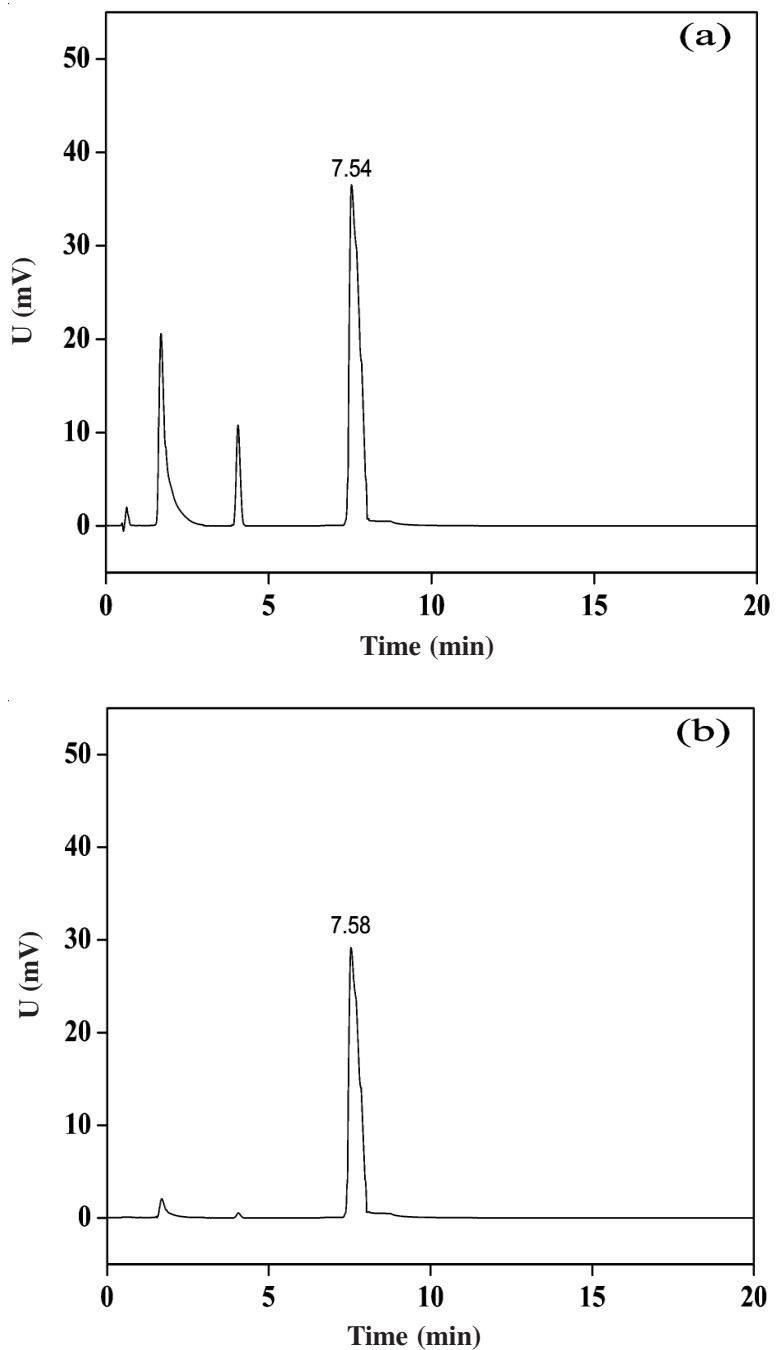

Fig. 7. HPLC chromatograms corresponding to (a) berberine sample and (b) elution solution after MIP-SPE of berberine sample 


\section{Conclusion}

The target molecularly imprinted polymers for berberine hydrochloride adsorption were successfully synthesized through the combination of ethylene glycol maleic rosinate acrylate as the cross-linker, berberine hydrochloride as the template and methacrylic acid as the functional monomer. The resulting molecularly imprinted polymers exhibited loose, porous structures. Scatchard analysis reveals the formation of two different types of binding sites in the molecularly imprinted polymers. The heat flow curve shows an endothermic peak and two relatively weak exothermic peaks for the adsorption process. The molecularly imprinted polymers exhibit highly selective adsorption for berberine hydrochloride as well as high stability and high regeneration capacity for berberine hydrochloride binding.

\section{ACKNOWLEDGEMENTS}

The authors acknowledged the National Natural Science Foundation of China (30960306 and 21065001), the Guangxi Natural Science Foundation of China (0991001Z) and the Guangxi New Century Hundred, Thousand and Ten Thousand Talent Project (No. 201292) for supporting this research work.

\section{REFERENCES}

1. M. Zayats, M. Kanwar, M. Ostermeier and P.C. Searson, Macromolecules, 44, 3966 (2011).

2. S.H. Cheong, S. McNiven, A. Rachkov, R. Levi, K. Yano and I. Karube, Macromolecules, 30, 1317 (1997).
3. W.G. Dong, M. Yan, Z. Liu, G.S. Wu and Y.M. Li, Sep. Purif. Technol., 53, 183 (2007).

4. N. Minoura, K. Idei, A. Rachkov, Y.W. Choi, M. Ogiso and K. Matsuda, Macromolecules, 37, 9571 (2004).

5. P.P. Qi, J.C. Wang, J. Jin, F. Su and J.P. Chen, Talanta, 81, 1630 (2010).

6. Q.J. Zhu, L.P. Wang, S.F. Wu, W. Joseph, X.H. Gu and J. Tang, Food Chem., 113, 608 (2009).

7. F.X. Liang, X.L. Zhu, J. Yang and Q.G. Su, Asian J. Chem., 20, 3954 (2008).

8. F. Tasselli, L. Donato and E. Drioli, J. Membr. Sci., 320, 167 (2008).

9. H.F. Wang, Y.Z. Zhu, J.P. Lin and X.P. Yan, Electrophoresis, 29, 952 (2008).

10. F. Rong, X.G. Feng, C.W. Yuan, D.G. Fu and P. Li, J. Liq. Chromatogr. R.T., 29, 2593 (2006)

11. V. Abbate, A.R. Bassindale, K.F. Brandstadt and P.G. Taylor, J. Catal,, 284, 68 (2011).

12. C.J. Stephenson and K.D. Shimizu, Polym. Int., 56, 482 (2007).

13. F.W. Wan, J.H. Yu, P. Dai and S.G. Ge, Anal. Lett., 43, 1033 (2010).

14. V.P. Joshi, S.K. Karode, M.G. Kulkarni and R.A. Mashelkar, Chem. Eng. Sci., 53, 2271 (1998).

15. L. Ye, R. Weiss and K. Mosbach, Macromolecules, 33, 8239 (2000).

16. H. Kim, K. Kaczmarski and G. Guiochon, Chem. Eng. Sci., 60, 5425 (2005).

17. I.S. Chronakis, B. Milosevic, A. Frenot and L. Ye, Macromolecules, 39, 357 (2006).

18. W. Yao, Y.J. Fang, G.L. Li, Z.X. Gao and Y.Y. Cheng, Polym. Adv. Technol., 19, 812 (2008).

19. V. Marthada, J. Res. NBS Stand., 85, 467 (1980).

20. X.L. Ma, Z. Chen, R.Y. Chen, X. Zheng, X. Chen and R.F. Lan, Polym. Int., 60, 1455 (2011).

21. L.N. Chen, X.J. Jia, Q. Lu, Y. Peng, S.H. Du and Q. Chen, Sep. Purif. Technol., 71, 324 (2010).

22. T. Takeuchi, T. Mukawa, J. Matsui, M. Higashi and K.D. Shimizu, Anal. Chem., 73, 3869 (2001). 